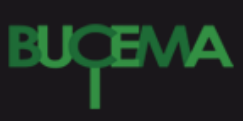

Bulletin du centre d'études médiévales d'Auxerre | BUCEMA

Hors-série $n^{\circ} 7 \mid 2013$

Les nouveaux horizons de l'ecclésiologie : du discours clérical à la science du social

\title{
Ecclesia y modelos de autoridad. Una reflexión a partir de las Sententiae de Isidoro de Sevilla y Tajón de Zaragoza (633-683)
}

Eleonora Dell'Elicine

\section{OpenEdition}

\section{Journals}

Edición electrónica

URL: https://journals.openedition.org/cem/12826

DOI: $10.4000 /$ cem. 12826

ISSN: 1954-3093

Editor

Centre d'études médiévales Saint-Germain d'Auxerre

Referencia electrónica

Eleonora Dell'Elicine, «Ecclesia y modelos de autoridad. Una reflexión a partir de las Sententiae de Isidoro de Sevilla y Tajón de Zaragoza (633-683) », Bulletin du centre d'études médiévales d'Auxerre I BUCEMA [En ligne], Hors-série $n^{\circ} 7$ | 2013, mis en ligne le 11 juin 2013, consulté le 02 mars 2023. URL : http://journals.openedition.org/cem/12826 ; DOI : https://doi.org/10.4000/cem.12826

Este documento fue generado automáticamente el 2 marzo 2023

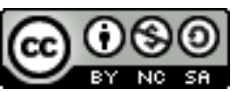

Creative Commons - Attribution - Pas d'Utilisation Commerciale - Partage dans les Mêmes Conditions 4.0 International - CC BY-NC-SA 4.0

https://creativecommons.org/licenses/by-nc-sa/4.0/ 


\title{
Ecclesia y modelos de autoridad. Una reflexión a partir de las Sententiae de Isidoro de Sevilla y Tajón de Zaragoza (633-683)
}

\author{
Eleonora Dell'Elicine
}

1 Tajón, obispo de Zaragoza entre 651 y c. 683, inicia de este modo el primer libro de sus Sententiarum libri $V$ :

«Caput Primum: Quod Deus incommutabilis, summus et aeternus existat. (S. Gregor, Moral., lib. XII, cap. 33, no 38). Solus Deus in semetipso incommutabilis est, quia solus habet immortalitatem : de quo per Jacobum dicitur: Apud quem non est transmutatio, nec vicissitudinis obumbratio (Jac. 1, 17) ${ }^{1}$.

2 Ya el género, título y contenido de este capítulo inaugural nos remiten sin equívoco a la obra de Isidoro, el gran obispo de Sevilla o, -como tan amorosamente anota el concilio VIII de Toledo del que Tajón participa, "nostri quoque doctor egregius " ${ }^{2}$ - muerto a esta altura más de quince años ha ${ }^{3}$. La relación entre ambos es todo menos casual : en efecto, este Tajón fue discípulo directo de Braulio, alumno dilecto- a su vez- del propio Isidoro.

3 Tanta credencial de ortodoxia, sin embargo, no ha propiciado en demasía el interés de los visigotistas por su obra. La escasa vocación por la novedad que nuestro autor ofrece, sumado al estilo de remisión enmarañada que muestran ya las cortas líneas escogidas de su texto- evocación de las ideas de Isidoro, de párrafos enteros de Gregorio Magno ${ }^{4}$, y de citas extraídas de la Biblia ${ }^{5}$ - ayudan a explicar la relegación que experimenta la obra de este obispo. Manuel Díaz y Díaz nos lo advierte en cuatro palabras : « un collage de citas », es la definición magistral que el filólogo gallego asesta en relación al estilo de Tajón ${ }^{6}$.

4 ¿Por qué Tajón, ilustre obispo de una de las ciudades más importantes del reino, ciñe su propia acción y pensamiento al molde de una obra producida una década y media atrás? ¿Qué persigue al retomarla, extenderla e infundirle desplazamientos que no figuraban 
de ese modo en el texto referencial? Nuestra hipótesis es que el zaragozano, en tiempos de monarquía fuerte, advierte la necesidad de consolidar el modelo de ecclesia diseñado por Isidoro, al tiempo que explora otros modelos de autoridad desactivados expresamente por el doctor sevillano en sus Sententiae. El propósito de este trabajo, en suma, es sopesar los cambios y ajustes producidos al proyecto isidoriano por el propio clero visigodo en la segunda mitad del siglo vII, e intentar explicarlos.

\section{Isidoro : sus Sententiae, las Moralia}

En su ya canónica edición de las Sententiae isidorianas, Pierre Cazier anotaba una serie de rarezas, de particularidades de esta obra en relación al resto de la producción del hispalense- su falta de prólogo, el empleo frecuente de vulgarismos, su plan poco claro, etc.; elementos que en conjunto llevaban al filólogo a considerar que se trataba de un texto con fines prácticos, unos apuntes prolijos de clase, el compendio último de la experiencia episcopal de Isidoro ${ }^{7}$.

6 Un punto más convendría añadir al catálogo ya largo de Cazier : la distancia que Isidoro toma en esta obra respecto a la gramática. Apartándose del consejo que Gregorio le había dirigido casi para él en contra del cultivo de esta disciplina en el prólogo de las Moralia ${ }^{8}$, Isidoro hasta el momento había considerado a la gramática el pivote del saber, una garantía de enunciado verdadero ${ }^{9}$. Esta es la disciplina que da el pie inicial a las Etimologías ${ }^{10}$; y en la definición que ofrece acerca de ella vuelve a enfatizar su lugar de privilegio ${ }^{11}$. Sin embargo, otra es la consideración acerca de la gramática que Isidoro hace en las Sententiae : si bien allí no descarta su utilidad ${ }^{12}$, advierte sobre los efectos engañosos de esta materia y desestima su empleo ${ }^{13}$. En términos prácticos, la operación permite advertir que en las Sententiae, Isidoro está pensando en unos modos de interpretación del mensaje bíblico más verticales- incluso en el interior del clero- que los postulados por él mismo en otras obras ${ }^{14}$.

7 En diversas cuestiones de igual relieve, el hispalense sólo sistematiza y refuerza lo que siempre en otros escritos ya había postulado: fundamentalmente el respeto por el orden jerárquico y un tratamiento precavido de las manifestaciones milagrosas.

8 En relación a lo primero y en la misma línea de lo que defendió en el concilio IV de Toledo, Isidoro impulsa la inclusión estable de la jerarquía eclesiástica en el círculo de la dirigencia visigoda ${ }^{15}$. De este modo, reyes, seniores y clérigos son congregados en Sent. III, 48, 7 dentro de una misma categoría englobadora : la de prelatii ${ }^{16}$. El expediente escogido por Isidoro para darle raíz a esta intervención es apoyarse en las Moralia de Gregorio. En una sentencia preparatoria, Isidoro entra en tema utilizando una palabra de contornos imprecisos, la de dignitas, y predica para ella conductas extraídas de un párrafo en donde el papa se refiere explícitamente sólo a la acción del clero ${ }^{17}$. Ya en el centro del problema, homologados los reyes y los hombres santos, el contenido de la sentencia se apoya con fuerza en la autoridad del papa magno ${ }^{18}$. Como podemos advertir, el hispalense manipula estratégicamente el texto de Gregorio : por momentos se aparta, en otros lo modifica, y en otros descansa en las proposiciones que ampara la autoría gregoriana.

9 Coherente con los postulados que esgrime, Isidoro defiende explícitamente el respeto al orden instituido ${ }^{19}$, la disciplina conveniente a cada grado eclesiástico ${ }^{20}$, la dignidad alcanzada $^{21}$. La eclesiología de Isidoro descansa primeramente en el rito de 
ordenación ${ }^{22}$. El cumplimiento correcto del mismo, unido al mantenimiento de la ética que el honor conlleva, asegura al resto de la grey un tránsito tranquilo y condiciones necesarias de salvación ${ }^{23}$.

10 Así como del primer al último de sus libros conserva la adhesión a los mecanismos y protocolos establecidos, otro tanto sostiene- como decíamos- respecto a la consideración del milagro. Sabemos que entre los visigodos es un rey- Sisebuto (612-621), por otra parte amigo y admirador a su contemporáneo Isidoro- quien inicia la tradición de recoger por escrito los milagros de un obispo fallecido poco tiempo atrás ${ }^{24}$. Por su parte también Gregorio Magno, preocupado por quienes sostenían que la falta de milagros en el presente indicaba una sustracción de la Gracia, se muestra partidario de dar a conocer los signos que patentizaban la vigencia de la promesa divina para con la Iglesia ${ }^{25}$. No es el caso de Isidoro, quien, perfectamente al corriente de estas posiciones, se atiene una vez más a la postura conservadora. Para el obispo de Sevilla, en época de los apóstoles los signos y milagros constituían una forma eficaz de difundir la fe entre los que todavía no creían; pero en los tiempos presentes, cuando la iglesia es dominante, esos signos se vuelven a su criterio innecesarios e inclusive peligrosos ${ }^{26}$. En efecto, considera que los portentos son señales del anticristo, y por lo tanto sólo medios de confusión para los probos ${ }^{27}$.

11 Con justeza Cazier señala que «Isidore semble insister sur la victoire finale des élus et de l'Église, plutôt que sur l'aspect terrible du jugement " ${ }^{28}$ : para el de Hispalis no hay que apurar los tiempos, los elegidos triunfarán a pesar de las tribulaciones, se trata de la segunda llegada del mesías. Esta escatología optimista, a nuestro modo de entender, se engarza bien con la eclesiología conservadora que venimos señalando en Isidoro : en efecto ¿A qué personaje se adjudicaría, en la práctica, la cualidad milagrosa? ¿Quién lo llevaría a cabo? ¿Y qué fines perseguiría con ello? Para el hispalense, la promoción de la santidad a través del milagro podía resultar, en los tiempos que corrían, una vía para granjearse un tipo de legitimidad alternativa a la que institución de lo sagrado establecía ; o para eludir, dentro de la institución, muchas de sus salvaguardas. Milagro e institucionalidad resultan, para el hispalense, dos opciones divergentes, casi incompatibles, en la agotadora tarea de mantener el orden.

\section{Tajón : sus Sententiae, las Moralia, las Sententiae de Isidoro}

12 ¿Y nuestro obispo Tajón? Como habíamos señalado, una década y media después de la puesta en circulación de las Sententiae, este conocedor profundo del texto bíblico ydebido a sus viajes y misiones en Roma- de la obra de Gregorio Magno, retoma el género y redacta un texto ya no en tres libros como lo había hecho Isidoro, sino en cinco largas partes.

13 La redacción de las Sententiae de Tajón es posterior a 652, fecha en que se llevaría a cabo la rebelión del tyrannus Froia- como lo califica Tajón- contra Recesvinto (653- 672), situación que impele a Tajón a tomar la pluma ${ }^{29}$.

Desde el punto de vista de los asuntos del regnum, muchas cosas habían cambiado desde los tiempos del viejo Isidoro. La posición dirigente del clero resultaba indiscutible, los privilegios de la silla toledana estaba encauzados, e incluso un monumental corpus de leyes, que contaba con la anuencia de Dios y de sus representantes en la tierra- el Liber 
Iudiciorum-, estaba a punto de ser promulgado ${ }^{30}$. Fronteras afuera, la situación también había girado, pero por caminos inquietantes : dos años después de la muerte de Isidoro, la ciudad santa- como sabemos- es arrancada de las ortodoxas manos de Heraclio para caer bajo el imperio de los infieles ; en 641 las huestes islámicas se hacen con Egipto ; en 644 con el Imperio Sasánida y en 651 han llegado a Jurasán ${ }^{31}$. Estos sucesos dramáticos son interpretados por judíos y cristianos en clave escatológica: aunque no resulta probable que las proposiciones de Pseudo Metodio hayan sido conocidas en occidente antes de $700^{32}$, profecías milenaristas o escatológicas en general capeaban sin más por los círculos expectantes de judíos y de cristianos del reino ${ }^{33}$.

En su Vitae sancti Aemilianii, Braulio de Zaragoza (631-651)- maestro de Tajón como ya apuntamos- fue el primer obispo visigodo que toma nota de la nueva proliferación de señales en la tierra ${ }^{34}$. Siguiendo esta vertiente, Tajón también admite la existencia de milagros en la iglesia presente. Escribe el zaragozano:

«(...) Omnipotens Deus in gradibus Ecclesiae agnoscitur, quia in singulis eius virtutibus atque miraculis, quam sit timendus et amandus, omnibus demonstratur: cum videlicet nunc suscipit, cum a culpis suis ad eum per poenitentiam redit (...). $»^{35}$.

A diferencia de lo que acabamos de observar con Isidoro, para el nuevo obispo de Zaragoza los milagros existen en el presente, y no son incompatibles con la institucionalidad sino que, por el contrario, los signos verdaderos sólo se producen en el interior de la institución eclesiástica. Así lo sigue afirmando en el libro siguiente :

«Sancta Ecclesia quotidie miracula spiritaliter facit, quod prisco tempore per sanctos apostolos corporaliter faciebat, sicut scriptum est: Signa autem eos qui credituri sunt, haec sequentur : in nomine meo daemonia ejicient, linguis loquentur novis, serpentes tollent, et si mortiferum quid biberint, non eis nocebit: super aegros manus imponent, et bene habebunt (Marc. XVI, 17). Sacerdotes Ecclesiae cum per exorcismi gratiam manum credentibus imponunt, et habitare malignos spiritus in eorum mente contradicunt, quid aliud faciunt nisi daemonia ejiciunt? $»^{36}$.

Como podemos advertir, son los ordenados los que para el zaragozano detentan en el presente la capacidad de producir signos.

En la mayor parte de estas sentencias, Tajón sigue de cerca el pensamiento de Gregorio, especialmente en las Moralia. En el caso de la primera cita [omnipotens Deus...], el zaragozano reenvía explícitamente a su lector a Moral. VIII, 48, 82 ; en donde el papa no está discurriendo acerca de las virtudes eclesiásticas, sino por el contrario está advirtiendo que aquellos que dentro de la iglesia ostenten los dones como si fuesen propios, serán más duramente castigados ${ }^{37}$. Lo que nos permite advertir esta remisión es que, en la superficie de su texto, Tajón está recuperando lo más potente de la producción milagrera- ese estar en la gracia que tanto desvelaba a los interlocutores de Gregorio-; pero que más profundamente, y reposando en la autoridad del papa magno, el de Zaragoza está advirtiendo a los díscolos acerca de los peligros de una desviación.

Estas nuevas Sententiae constituyen un texto exhortativo, una amonestación remozada, extendida esta vez a cinco libros debido a lo perentorio de la situación escatológica.

El prefacio dedicado a su colega Quirico de Barcelona nos vuelve a colocar en esta pista interpretativa. Allí Tajón describe los efectos que ha dejado en Zaragoza la invasión de los vascones empujada por el tirano Froia :

«(...) Heu, pro dolor! (..) Innoxius quippe multorum Christianorum sanguis effunditur: alii jugulis, nonnulli missilibus, plerique diversis jaculis sauciantur, 
innumerabilis multitudo captivorum abducitur, inmensa spolia subtrahuntur. Templis Dei infaustum bellum infertur, sacra alteris destruuntur, plerique ex clericats officio easibus obtruncantur, atque inhumata canibus avibusque multorum exponuntur cadavera occisorum : ita ut septuagesimi octavi psalmi non immerito illi ea calamitati congrua videatur inscriptio ${ }^{38}$.

Nuevamente el texto anuda dos planos : el registro de lo que efectivamente ha sucedido y el de su eminente castigo. La remisión al salmo 78 nos avisa que son malditos los bárbaros que han propinado tamaña desgracia en la heredad del Señor- y con ellos todos los que los han incitado ${ }^{39}$. Hay bárbaros que desconocen el nombre de Dios, pero entre los que los conocen, elegidos y réprobos no tendrán el mismo destino. Repitiendo el gesto de sus homólogas isidorianas, las Sententiae de Tajón promueven una contrición del corazón en el marco estricto del respeto a las jerarquías y a la ortodoxia. Realizan un llamamiento a replegarse a la institución, a la tradición signada por los Padres, a las orientaciones nodales señaladas por Isidoro. Esta exhortación a respetar lo que hay, no obstante, se enriquece con el condimento de nuevas dimensiones : a una autoridad que se legitima a través del rito y de las obras; Tajón le añade la posibilidad que ostente carisma en el marco siempre reglado de la institución. Más de quince años después de la muerte del maestro, en suma, la solución pensada por el obispo de Zaragoza es la de institucionalizar los dones.

\section{Conclusiones}

En tiempos en que proliferan- como lo demuestran las reglas de los padres y la propia arqueología- soluciones ligadas al eremitismo y a la ruptura de los lazos instituidos, esta obra del obispo Tajón nos muestra sin embargo un llamado a habitar, a poner cuerpo a lo cimentado y garantido por la tradición legada por Isidoro.

Mediando el siglo vII, el modelo isidoriano goza todavía de salud, creatividad y vigencia. Sin embargo- y como nos indican la experiencia casi contemporánea de Fructuoso de Braga e incluso la de Valerio del Bierzo- esto no refleja la diversidad de las prácticas sociales, sino que debemos entenderlo como una propuesta que lucha vigorosamente por sostener su función cohesiva.

\section{NOTAS}

1. taio caesaraugustanus, Sententiarum libri quinque, I, 1, PL LXXX, col. 751, complementado con E. ANSPACH, Taionis et Isidori. Nova fragmenta et opera, Centro de Estudios Históricos, Madrid, 1930.

2. «... ecclesiae catholicae novissimum decus, praecedentibus aetate postremus, doctrinae comparatione non infimus, et, quod maius est, in saeculorum fine doctissimus atque cum revertentia nominandus, Isidorus ... » Conc. Tol. VIII, 499-502, in G. MARTÍNEZ DIEZ, F. RODRÍGUEZ, La colección canónica hispana V. Concilios Hispanos, II parte, CSIC, Madrid, 1992.

3. Efectivamente, de este modo comienzan las Sentencias de Isidoro, que Tajón resignifica en el primer capítulo de las suyas : «I : Quod Deus summus est et incommutabilis sit : Summum bonum Deus est, quia incommutabilis est et corrumpi omnino non potest. Creatura vero bonum, sed non summum 
est, quia mutabilis est ut, dum sit quidem bonum, non tamen esse potest et summum. " Isidorus Hispalensis, Sent. I.1, in P.CAZIER, Isidorus Hispalensis Sententiae, Turnhout, 1998. Para el alineamiento de Tajón en relación a Isidoro, E. CONDE GUERRI, « Tajón de Zaragoza y su tradición doctrinal sobre los pastores animarum », in Aragón en la Edad Media 14-15, 1 (1999) p. 329-339.

4. «Ecce inter sanctos eius nemo immutabilis, et caeli non sunt mundi in conspectu eius : Hoc caelorum nomine repetiit quod sanctorum prius appelatione signavit. Nam de eisdem sanctis scriptum est : Caeli enarrant gloriam Dei. Qui per naturam omnes in semetipsis propiam mutabilitatem habent, sed dum immutabili veritati studiose semper inhaerere desiderant, inhaerendo agunt ut immutabiles fiant. Cumque ad hanc toto affectu se tenent, quandoque accipiunt ut super semetipsos ducti vincant hoc quod in semetipsis mutabiles exsisterunt. Quid enim mutabilitas nisi mors quaedam est? Quae dum rem quamlibet in aliam immutat, quasi occidit quod fuerat, ut incipiat esse quod non erat. Et de auctore omnium scriptum est: Qui solus habet immutabilitatem, quia videlicet in semetipso solus immutabilis est. De quo per Iacobum dicitur: Apud quem non est transmutatio, nec vicissitudinis obumbratio. Ipsa enim mutabilitas umbra est, quae quasi obscuraret lucem, si hanc per aliquas vicissitudines permutaret. Sed quia in Deo mutabilitas non venit, nulla eius lumen umbra vicissitudinis intercidit. Bene autem dicitur: Caeli non sunt mundi in conspectus eius, quia per semetipsos ante districtum Dei iudicium nec ipsi esse mundi ad perfectum possunt qui munditiae praedicatores fiunt; Ioanne attestante, qui ait: Si dixerimus quia peccatum non habemus, ipsi nos seducimus. Si igitur inter sanctos illius nemo immutabilis est, et caeli in conspectus illius non sunt mundi, quis apud se de iustitiae opera praesumat? » Greg., Moral. XII, 33, 38. Para un análisis técnico del uso de citas de Gregorio en otro de los textos de Tajón A. PALACIO MARTíN, « Tajón de Zaragoza y la explicatio » In cantica canticorum, in Anuario de Estudios filológicos, 3, 1980, p. 115-127.

5. La cita completa del versículo citado por Tajón en nuestra nota 1 es : « omne datum optimum et omne donum perfectum desursum est descendens a Patre luminum apud quem non est transmutatio nec vicissitudinis obumbratio ». Ep. Jac. 1, 17.

6. «La sua illimitata ammirazione per Gregorio lo portò pure a comporre una sintesi di tutta la dottrina cristiana mediante la raccolta di frasi e massime gregoriane, imitando in parte, ma con menore originalità, tecnica e struttura, le Sentenze di Isidoro, seguendole da vicino, ma con una nuova concezione delle massime, basata qui sulla semplice tecnica del collage. Così realizza $i$ Sententiarum libri V, che ebbero no poca diffusione. Col precedente isidoriano, Taione si propose di documentarne tutta la teologia con frasi estratte quasi exclusivamente dalle opere di Gregorio, in particolare dai Moralia. » ; M.DÍAZ Y DíAz, «Scrittori della peninsola iberica», in A. DI BERNARDINo, Patrologia. I patri latini (sec. V- VIII), Institutum Patristicum Agustinianum, Marietti, Génova, 1996, p. 108. Comentario semejante es el de Ursino Domínguez : « Aunque nada tiene de original, fuera del orden en que dispuso los textos de san Gregorio, por ser uno de los primeros (el segundo, pues lo precedió Isidoro) en este género de composiciones que tanto imitaron después los escolásticos, y en particular Pedro Lombardo, merece especial atención y estima. » U. DOMÍNGUEZ DEL VAL, « Escritores españoles », in B. ALTANER, E. CueVAS, U. Domínguez del VAL, Patrología, Espasa- Calpe, Madrid, 1962, p. 514-515. Nota breve de esta obra in E. DEKKERS, Clavis Patrum Latinorum, CCSL, Turnhout, 1995, p. 442.

7. "Quoi qui'il en soit même si l'on ne sait pas exactement à quel moment chacune des sentences a été composée, on peut dire avec certitude qu'elles sont le couronnement de toute la vie d'Isidore, de tout son enseignement pastoral, et aussi son oeuvre la plus personnelle, celle qui traduit le mieux son projet d'évèque pour l'Église de son temps, en quelque sorte, son testament spirituel, puisqu'il devait mourir en 636, trois ans après le IVème. Concile de Tolède » $\mathrm{P}$. CAZIER, "Introduction", in Isidorus Hispalensis sententiae, Turnhout, 1998, p.XIX. Las particularidades señaladas figuran en p.XVII, XVIII, XXXIII especialmente.

8. En efecto, Gregorio escribía lo siguiente en su prefacio a las Moralia dirigidas a Leandro : «(...) Quaeso, autem, ut huius operis dicta percurrens in his verborum folia non requiras, quia per sacra eloquia ab eorum tractatoribus infructuosae loquacitatis levitas studiose compescitur, dum in templo Dei nemus 
plantari prohibetur. Et cuncti procul dubio scimus quia, quoties in foliis male laetae segetis culmi proficiunt, minori plenitudine spicarum grana turgescunt. Unde et ipsam loquendi artem, quam magisteria dicipliae exterioris insinuant, servare despexi. Nam sicut huius quoque epistolae tenor enuntiat, non metacismi collisionem fugio, non barbarismi confusionem devito, situs modosque et praepositionum casus servare contemno, quia indignum vehementer existimo, ut verba caelestis oraculi restringam sub regulis Donati » Greg. Magn., Moral., Ep. Ded. 5, 207-222 in M. ADRIAEN (ed.), S. Gregorii Magni. Moralia in Job, CCL CXLIII, a/b, Turnhout, 1979-1985. También se ha trabajado de modo simultáneo con la edición de R. GILLET, A. DE GAUDEMARIS, (eds.), Grégoire le Grand. Morales sur Job, Paris, 1950.

9. A propósito de este punto, dice Fontaine : «La place d'honneur que tiennent Augustin et Cassiodore parmi les "auctores" d'Isidore de Séville apparaîtra clairement (...). Mais il ressort dès maintenant qu'ils ont été pour lui de solides garants de la légitimité du savoir grammatical. C'est à eux qu'il doit sans doute de n'avoir pas été impressioné par les critiques que l'ami de son frère Léandre, le pape Grégoire, avait adressées à la grammaire dans l'épittre d'envoi des Moralia in Job qu'il adressa à ce même Léandre en 595 »: J. FONTAINE, Isidore de Séville et la culture classique dans l'Espagne visigothique, t. I., éd. Agustiniennes, Paris, 1959, p. 33 y ss. Más adelante agrega: "La grammaire est "origine" des disciplines, comme l'étymologie est "origine" des vocables. (...). Elle [la grammaire] devient un système complet d'explication du monde a fortiori de la philosophie elle- même. ", ibid., p. 53-54. Sobre este tema, también J. FONTAINE, "Grammaire sacrée et grammaire profane: Isidore de Séville devant l'exégèse biblique ", Antigüedad y cristianismo III, 1986; J. FONTAINE, Isidoro de Sevilla. Génesis y originalidad de la cultura hispánica en tiempos de los visigodos, Encuentro, Buenos Aires, 2002 ; I. VELÁzQUEZ, Latine dicitur, vulgo vocant. Aspectos de la lengua escrita y hablada en las obras gramaticales de Isidoro de Sevilla, Fundación san Millán de la Cogolla, Logroño, 2003, particularmente p.47-61; M. DÍAZ Y DÍAZ, Enciclopedismo e sapere cristiano tra tardo-antico $e$ alto medioevo, Milan, 1999, especialmente p. 109 y ss; y más general J. A. PUENTES ROMAY, «Lengua y gramática en el occidente peninsular altomedieval. Algunas consideraciones generales » in Actas II congreso hispánico de latín medieval (León, 11- 14 de noviembre de 1997), vol. II, Universidad de León, León, 1998.

10. «Disciplinae liberalium artium septem sunt. Prima grammatica, id est loquendi peritia. Secunda rhetorica, quae propter nitorem et copiam eloquentiae suae maxime in civilibus quaestionibus necessaria existimatur. Tertia dialectica cognomento logica, quae disputationibus subtilissimis vera secernit a falsis. Quarta aritmetica, quae continet numerum causas et divisiones. Quinta musica, quae in carminibus cantibusque consistit. Sexta geometrica, quae mensuras terrae dimensionesque conplectitur. Septima astronomia, quae continet legem astrorum. » Isid. Hisp., Etym. I, 2, 1; in J. OROZ RETA, M. MARCOS CASQUERO, San Isidoro de Sevilla. Etimologías I, BAC, Madrid, 1982.

11. "Grammatica est scientia recte loquendi, et origo et fundamenta liberalium litterarum. Haec in disciplinis post litteras communes inventa est, ut iam qui didicerant litteras per eam recte loquendi rationem sciant. ». Isid. Hisp., Etym. I, 5,1.

12. "Meliores esse grammaticos quam hereticos; heretici enim haustum letiferi sucus hominibus persuadendo propinant; grammmaticorum autem doctrina potest etiam proficere ad vitam, dum fuerit in meliores usus adsumpta. » Isid. Hisp., Sent. III, 13, 11.

13. 13, 9: "Nihil aliud agit amor mundanae scientiae, nisi extollere laudibus hominem. Nam quanto maiora fuerint litteraturae studia, tanto animus arrogantiae fastu inflatus, maiore intumescit iactantia. Unde et bene psalmus ait: Quia non cognovit litteraturam, introibo in potentias Domini ». 13. 10 : «Simplicioribus litteris non est proponendus fucus grammaticae artis. Meliores sunt enim communes litterae quia simpliciores, et ad solam humilitatem legentium pertinentes; illae vero nequiores quia ingerunt hominibus perniciosam mentis elationem. » Isid. Hisp., Sent. III, 13, 9 ; 13, 10.

14. Velázquez resalta la preocupación de Isidoro: «Pero son, sobre todo, aquellos pasajes que veíamos a propósito de los Synonyma y también de las Sententiae, los que ponen de manifiesto esta preocupación constante y doble, la correcta formación y preparación del clero y los predicadores 
a través del studium y muy especialmente del mejor de los instrumentos posibles para ello, la lectura de las Sagradas Escrituras, y la adaptación de estos lectores formados ante el gran público al que han de instruir en el mensaje divino y en la forma de vida » : I. VeLÁZquEZ, Latine dicitur, vulgo vocant... op. cit., p. 201.

15. Los mecanismos conciliares a través de los cuales Isidoro buscó esta inclusión fueron principalmente dos: la institucionalización anual de un concilio general (artículo IV) y la apelación judicial a los obispos (artículo XXXII).

16. "Reges a recte agendo vocati sunt, ideoque recte faciendo regis nomen tenetur, peccando amittitur. Nam et viros sanctos proinde reges vocari in sacris eloquiis, eo quod recte agant, sensusque proprios bene regant et motus resistentes sibi rationabili discretione conponant. Recte igitur illi reges vocantur qui tam semetipsos quam subiectos, bene regendo, modificare noverunt. » Isid. Hisp. Sent. III, 48, 7. Cf. P. CAZIER, «Introduction », op. cit., p. XXIX.

17. Cita de Isidoro: "Quanto quisque amplius saecularis honoris dignitate sublimatur, tanto gravius curarum ponderibus adgravatur, eisque magis mente et cogitatione subicitur, quibus sublimitatis gradu praeponitur. Nam ut quidam patrum ait: Omne quod supereminet, plus maeroribus afficitur quam honoribus gaudet." Isid. Hisp., Sent. III, 48, 3. Cita de Gregorio: "Alius pensare pondus honoris ecclesiastici neglegens, ad locum regiminis praemiis ascendit. Sed quia omne quod hic eminet plus maeroribus afficitur quam honoribus gaudet, dum cor tribulatione premitur, ad memoriam culpa revocatur; doletque se ad laborem cum culpa pervenisse, et quam sit iniquum quod admiserit ex ipsa fractus dificultate cognoscit. (...) 》 Greg. Magn., Moral. XXXII, XX, 38, 54/59.

18. La cita de Isidoro que corresponde es Sent. III, 48,7 ya trabajada. La de Gregorio evocada es la siguiente: "Bene autem sancti viri scripturae sacrae testimonio reges vocantur, quia praelati cuntis motibus carnis, modo luxuriae appetitum frenant, modo aestum avaritiae temperant, modo gloriam elationis inclinant, modo suggestionem livoris obruunt, modo ignem furores extinguunt. Reges ergo sunt, quia temptationum suarum motibus non consintiendo succumbere, sed regendo praeesse noverunt. Quia igitur ab hac potestate regiminis ad potestatem transeunt retributionis, dicatur recte: Reges in solio collocat in perpetuum. Semetipsos enim regendo, ad tempus fatigati sunt, sed in regni erectionis internae solio in perpetuumm collocantur; et eo illic accipiunt alios digne iudicare, quo hic nesciunt sibimetipsis nequiter parcere. Hinc enim alias dicitur: Donec iustitia convertatur in iudicium. Paulus de se suisque consortibus dicit: Ut nos efficeremur iustitia Dei in ipso. Iustitia ergo in iudicium vertitur, quia hi qui nunc iuste atque irreprehensibiliter vivuunt, tunc iudicandi potentiam nanciscuntur. Hinc Laodiceae Ecclesiae Dominus dicit : Qui vicerit, dabo ei sedere mecum in trhono meo, sicut et ego vici, et sedi cum Patre meo in throno eius. Vincens Dominus in throno cum Patre sedisse se asserit, quia post passionis certamina, post resurrectionis palmam, clarius se omnibus quod potestati Patris esset aequalis indicavit, eique se non disparem calcato mortis aculeo innotuit. Unde et Mariae necdum se credenti Patri similem, dicit: Noli me tangere, nondum enim ascendi ad Patrem meum. Nobis enim in throno filii sedere, est ex eiusdem Filii potestate iudicare. Quia enim iudicii principatum ex eius virtute percipimus, velut in eius throno residemus. Nec abhorret a vero quod alias super duodecim thronos venturos testatur esse discipulos, hic vero in throno duo perhibet esse sessuros. Per thronos, quippe duodecim universale iudicium, per thronum vero Filii singulare culmen iudiciariae potestatis ostenditur. Hoc ergo duodecim thronis, quod uno throno Filii designatur, quia videlicet univesale iudicium ex Mediatoris nostri interventione percipitur. Dicatur ergo : Reges in solio collocat in perpetuum. » Greg. Magn., Moral. XXVI, 28, 53.

19. "Propter peccatum primi hominis humano generi poena divinitus inlata est servitutis, ita ut quibus aspicit non congruere libertatem, his misericordius inroget servitutem. Et licet per peccatum humanae originis, tamen aequus Deus ideo discrevit hominibus vitam, alios servos constituens, alios dominos, ut licencia male agendi servorum potestate dominantium restringatur. Nam si omnes sine metu fuissent, quis esset qui a malis quempiam cohiberet? Inde et in gentibus principes regesque electisunt, ut terrore suo populos a malo coercerent, atque ad recte vivendum legibus subderent. » Isid. Hisp., Sent. III, 47, I. Para 
sopesar el grado de coherencia con otros trabajos de Isidoro, cf. Tol. IV, 75, particularmente 91-121.

20. «Multis intercipit Satanas fraudibus eos qui vitae et sensus utilitate praestantes, praeesse et prodesse aliis nolunt, et, dum eis regimen animarum inponitur, rennuunt, consultius arbitrantes otiosam vitam degere quam lucris animarum insistere. Quod tamen decepti agunt per argumentum diaboli fallentis eos per speciem boni, ut, dum illos a pastorali officio retrahit, nequaquam proficiant qui forum verbis atque exemplis instrui poterant. » Isid. Hisp., Sent. III, 33,2. A manera de ejemplo para sopesar el grado de coherencia con otros trabajos de Isidoro, cf. Tol. IV, 27, 39, etc.

21. "Plerique sacerdotes suae magis utilitatis causa quam gregis preaeesse desiderant, nec ut prosint praesules fieri cupiunt, sed magis ut divites fiant, et honorentur. Suscipiunt enim sublimitatis culmen non pro pastorali regimine, sed pro solius honoris ambicione, atque abiecto opere dignitatis, solam nominis appetunt dignitatem. » Isid. Hisp., Sent. III, 34,5. Para calibrar el grado de coherencia con otros trabajos de Isidoro, cf. Tol. IV, 20.

22. Cf. Tol. IV, 19 y 28.

23. "Sancti viri nequaquam occupationum saecularium curas appetunt, sed occulto ordine sibi superinpositas gemunt, et quamvis illas per meliorem intentionem fugiant, tamen per subditam mentem portant. Quas quidem summopere, si liceat, vitare festinant, sed timentes occultam dispensationem Dei, suscipiunt quod fugiunt, exercent quod vitare noscuntur. Intrant enim ad cor, et ibi consulunt quid velit occulta voluntas Dei: seseque subditos debere esse summis ordinationibus cognoscentes, humiliant cervicem cordis iugo divinae dispensationis. » Isid. Hisp., Sent. III, 33,3.

24. Se trata de la Vita Desiderii, redactada hacia 613. Observemos al punto un ejemplo de cómo el rey releva uno a uno los milagros ocurridos poco tiempo atrás : « De sus milagros [de Desiderio] he decidido seleccionar tres, aun cuando mi sobrio estilo se resienta por la falta de técnica. En cierta ocasión vino a visitarle un gran gentío. Ordenó él que, como es costumbre, les sirvieran de comer y de beber. Un sirviente le comunicó que la presencia del vino, lo más solicitado, era lo que más les había faltado. El recipiente en el que se había consumido la bebida mandó que se lo mostraran. Hecha la señal de la cruz, por la gracia del Salvador, se volvió a llenar con la generosidad de un vino excelente. De este modo la muchedumbre que acudió se restableció con la bendición al tiempo que con la mística bebida. » Sis., Vita Des., 12, in M. DÍAZ Y DíAz, (ed.), « Tres biografías latino medievales de san Desiderio de Viena. Traducción y notas », Fortunatae, 5 (1993). El milagro, como vemos, está rodeado de todas las señales del mando : la presencia del sirviente, la orden, el cumplimiento de lo solicitado por parte de la divinidad. Advirtamos de paso cómo semantiza directamente apoyándose en el relato de las bodas de Canaan (Ver Jn. 2, 1- 12). Para esta vita, J. FONTAINE, "King Sisebut's Vita Desiderii and the Political function of Visigothic Hagiography ", in E. JAMES (comp.), Visigothic Spain. New approaches, Oxford, 1980 ; J.C. MARTIN, «Caracterización de personajes y tópicos del género hagiográfico en la Vita Desiderii de Sisebuto », in Helmantica XLVIII, 147 (1997), p. 111-133.

25. «Petrus: Vellem quaerenti mihi de eis aliqua narrarares, neque hac pro re interrumpere expositionis Studium grave videatur, quia non dispar aedificatio oritur ex memoria virtutum. In expositione quippe qualiter invenienda atque tenenda sit virtus agnoscitur, in narratione vero signorum congnoscimus inventa ac retenta qualiter declaratur. Et sunt nonnulli quos ad amorem patriae caelestis plus exempla quam praedicamenta succedunt. Fit vero plerumque in audientis animo duplex adiutorium in exemplis partum, quia et ad amorem venturae vitae ex praecedentium conparatione accenditur, et iam si se esse aliquid aestimat, dum de aliis meliora cognoverit, humiliatur. Gregorius: Ea quae mihi sunt virorum venerabilium narratione conperta incunctanter narro sacrae auctoritatis exemplo, cum mihi luce clarius constet quia Marcus et Lucas evangelium quod scripserunt, non visu sed auditu didicerunt. Sed ut dubitationis occasionem legentibus subtraham, per singula quae describo, quibus mihi haec auctoribus sint conperta manifesto. Hoc vero scire te cupio quia in quibusdam sensum solummodo, in quibusdam vero et verba cum sensu teneo, quia si de personis omnibus ipsa specialiter et verba tenere voluissem, haec rusticano usu prolata stilus scribentis non apte susciperet. Seniorum valde venerabilium didici relatione 
cum narro. » Greg. Magn., Dial., LI, 9-10, in DE VOGÜE, P. ANTIN, (eds), Grégoire le Grand, Dialogues, t. II, Paris, 1979.

26. «De sanctorum miraculis : Etsi apostolis virtus data est signorum propter fidem gentium nutriendam, eccelsiae tamen data est virtus operum pro eandem fidem ornandam, et tamen in ipsis apostolis plus erat mirabilis virtus operum quam virtus signorum. Ita nunc et in ecclesia plus est bene vivere quam signa facere. » Isid. Hisp., Sent. I, 24,1.

27. «Ob hanc utilitatem cessabunt sub Antichristo ab ecclesia miracula et virtutes, ut per hoc sanctorum clareat patientia et reproborum qui scandalizabuntur levitas ostendatur, et persequentium audacia ferocior efficiatur. » Isid. Hisp., Sent. I, 24, 4b.

28. P. CAZIER, «Introduction », op. cit. p. XXIV.

29. "Optime novit, beatitudo vestra tempos illud quo tortuosus anguis ore pestifero in quorumdam mentibus virulenta seminum suorum sparserat zizania, fraudulentaque decepctione a tramite recti itineris gressum removerat mentium perditarum: in quo quidam homo pestifer atque insani capitis Froja tyrannidem sumens, assumptis sceleris sui perversis fautoribus, adversus orthodoxum magnumque Dei cultorem Recesvinthum principem fraudulenta praetendens molimina, superbo adnisu Christianam debellaturus aggreditur patriam. Hujus itaque sceleris causa gens esfera Vasconum Pyrenaeis montibus promota, diversis vastationibus Hiberiae patriam copulando crassatur. " Taio Caes, Sent., Praef. 2, col. 727, in PL LXXX, 727-990. Respecto a la fecha de redacción de la obra, Vega sostiene que es bastante posterior a la revuelta, cf. A. VEGA, «Tajón de Zaragoza. Una obra inédita », in España Sagrada 56 (1957), p.247. Ver para esta obra también C. DEL VALLE, « Tajón de Zaragoza (ca. 600-680) » in La controversia judeo-cristiana en España (desde los orígenes hasta el siglo XIII), CSIC, Madrid, 1998, p. 113. Sobre el reinado de Recesvinto y la rebelión de Froja J. ORLANDIS, Historia de España, época visigoda (409-711), Gredos, Madrid, 1987, p. 158. Aparentemente, Eugenio I de Toledo (muerto en 657) también hace una alusión a esta rebelión en su Carmen 5b (muy fragmentado) y también a la paz restablecida en el 36 en términos parecidos a los que formula Tajón [cf. P. ALBERTO (ed.) Eugenii Toletani opera omnia, CCSL CXIV, Turnhout, 2005. Para el paralelo, ver p. 16 de la introducción].

30. Al interior del reino, las administraciones de Chindasvinto (642-653) y su hijo Recesvinto (653-672) resultaron eficaces a la hora de sostener una política centralista en provecho de la monarquía : la sucesión filial, la promulgación inminente de un código monopólico de leyes y la serie de medidas en contra de los judíos son índices claros de esta reorganización. Para esta coyuntura, resultan especialmente luminosos los artículos de C. MARTIN, «La réforme visigothique de la justice : les années Recceswinth», in N. GUGLIELMI, A. RUCQUOI, Derecho y justicia : el poder en la Europa medieval, CNICT/CNRS, Buenos Aires, 2008 ; C. MARTIN, «Liber Iudiciorum », in Mélanges de la Casa de Velázquez. Nouvelle série, 41/2 (2011). Un panorama fáctico in P. DÍAZ MARTÍNEZ, C. MARTÍNEZ MASA, F. SANZ HUESMA, Hispania tardoantigua y visigoda, Madrid, 2007, p. 422 y ss.

31. Un panorama fáctico operativo in A. DUCELLIER, M. KAPLAN, B. MARTIN, De los bárbaros a los otomanos. El cercano oriente medieval, Madrid, Akal, 1988, p. 50 y ss. ; C. HILLENBRAND, « Muhammad and the rise of Islam », in P. FOURACRE (ed.), The new Cambridge medieval history, vol. I (500- 700), Cambridge, 2005, p. 317-345 ; R. collins, Early Medieval Spain. Unity in diversity, 400-1000, Hampshire, 1995, p. 146 y ss. ; H. KENNEDY, « The mediterranean frontier : Christianity face to face with Islam (600-1050) », in T. NOBLE, J. SMITH, The Cambridge History of Christianity. Early Medieval Christianities (600-1100), Cambridge, 2008; M. HoDson, The Venture of Islam. Conscience and History in a World Civilization, Chicago-London, 1974, p. 146-230, entre otros.

32. J. HILlgaRTH, «Escathological and political Concepts ", in J. FONTAINE, J. HILLGARTH, The seventh century, change and continuity, University of London, Londres, 1992, p. 228.

33. Para el pensamiento escatológico en el siglo VII especialmente L. SCHIFFMAN, « Messianism and apocalypticism in rabbinic texts ", in S. KATZ, (ed.), The Cambridge History of Judaism, Volume IV : the Late Roman-Rabbinic period, Cambridge, 2008 ; P. DE MARTIN DE VIVIÉs, Apocalypses et cosmologie du 
salut, París, 2002 ; J. VANDERKAM, W. ADLER, The Jewish apocalyptic heritage in early Christianity, AssenMinneapolis, 1996 ; R. LANDES, « Lest the millenium be fulfilled : apocalyptic expectations and the pattern of western cronography (100-800 CE)», in W. VERBEKE, D. VERHELST, A. WELKENHEYSEN, The use and abuse of Escathology in the Middle Ages, Leuven, 1988 ; R. LANDES, «Millenarismus absconditus. L'historiographie augustinienne et le millénarisme du haut Moyen Âge jusqu'à l'an Mil » in Le Moyen Âge, $\mathrm{n}^{\mathrm{o}}$ 3-4, t. XCVIII, 1992. Su conexión con el reino visigodo J. GIL, «Judíos y cristianos en la Hispania del siglo VII", in Hispania Sacra 30 (1977); C. MARTIN, La géographie du pouvoir dans l'Espagne visigothique, Paris, 2003 ; L. GARCÍA MORENo, Los judíos de la España antigua. Del primer encuentro al primer repudio, Madrid, 1993. L. GARCÍA MORENO, «Expectativas milenaristas y escatológicas en la España tardoantigua », in Arqueología, Paleontología y Etnografía, 4 (1997); B. DUMÉZIL, Les racines chrétiennes de l'Europe. Conversion et liberté dans les royaumes barbares ( $\mathrm{V}^{\mathrm{e}}$ $\mathrm{VIII}^{\mathrm{e}}$ siècle), Paris, 2005, p. 294 y ss.

34. «Volo autem ut, quia sanctissimus vir Cinonatus presbyter atque Gerontius adhuc in corpore degent, omnia quae in eo conscripsi ante ipsi recognoscant, et eorum discussione ventilata, si nec nominum nec rerum me fefellit sententia, habeantur confirmata. Sane illa quae anno praeterito a vobis ibidem divinitus operata didici, in finem libelli istius ut a vobis accepi adieci. » Br. Caes., Vit. Aem. I, in J. Oroz (ed.), Sancti Braulionis Caesaraugustani episcopi, Vita santi Aemiliani, Perficit IX, no 119-120; nov- dic. 1978. Observemos que los testigos principales son miembros ellos también de la iglesia.

35. Taio Caes., Sent. II, 12, col. 792.

36. Taio Caes., Sent. III, 17, col. 870.

37. « (...) O miseri qui affectantes laudes hominum, in semetipsis dissipant fructus laborum, cumque se ostendere alienis oculis appetunt, damnant quod agunt! Quos nimirum maligni spiritus cum ad iactantiam provocant, eorum sicut diximus, opera captivantes denudant. Unde sub cuiusdam gentis specie antiquorum hostium malitiam signans per prophetam Veritas dicit: Possuit vineam meam in desertum, decorticavit ficum meam, nudans exspoliavit eam, albi facti sunt rami eius. (...).» Greg. Magn. Moral. VIII, 48, 82.

38. Taio Caes., Sent., Praef., 2, col. 727-28.

39. «salm 78:1 psalmus Asaph Deus venerunt gentes in hereditatem tuam polluerunt templum sanctum tuum posuerunt Hierusalem in pomorum custodiam 2 posuerunt morticina servorum tuorum escas volatilibus caeli carnes sanctorum tuorum bestiis terrae 3 effuderunt sanguinem ipsorum tamquam aquam in circuitu Hierusalem et non erat qui sepeliret 4 facti sumus obprobrium vicinis nostris subsannatio et inlusio his qui circum nos sunt 5 usquequo Domine irasceris in finem accendetur velut ignis zelus tuus 6 effunde iram tuam in gentes quae te non noverunt et in regna quae nomen tuum non invocaverunt 7 quia comederunt Iacob et locum eius desolaverunt 8 ne memineris iniquitatum nostrarum antiquarum cito anticipent nos misericordiae tuae quia pauperes facti sumus nimis 9 adiuva nos Deus salutaris noster propter gloriam nominis tui Domine libera nos et propitius esto peccatis nostris propter nomen tuum 10 ne forte dicant in gentibus ubi est Deus eorum et innotescat in nationibus coram oculis nostris ultio sanguinis servorum tuorum qui effusus est 11 introeat in conspectu tuo gemitus conpeditorum secundum magnitudinem brachii tui posside filios mortificatorum 12 et redde vicinis nostris septuplum in sinu eorum inproperium ipsorum quod exprobraverunt tibi Domine 13 nos autem populus tuus et oves pascuae tuae confitebimur tibi in saeculum in generationem et generationem adnuntiabimus laudem tuam ». 
ÍNDICE

Mots-clés: société wisigothique, ecclésiologie, autorité

\section{AUTOR}

\section{ELEONORA DELL'ELICINE}

Universidad de Buenos Aires, Universidad de General Sarmiento 